\title{
Hand Control With Invasive Feedback Is Not Impaired by Increased Cognitive Load
}

\author{
Giacomo Valle1,2,3†, Edoardo D'Anna', Ivo Strauss 1,2, Francesco Clemente ${ }^{2,4}$, \\ Giuseppe Granata 5 , Riccardo Di lorio ${ }^{5}$, Marco Controzzi ${ }^{2,4}$, Thomas Stieglitz ${ }^{6}$, \\ Paolo M. Rossini7, Francesco M. Petrini ${ }^{1,3}$ and Silvestro Micera ${ }^{1,2 \star}$
}

\begin{abstract}
${ }^{1}$ Bertarelli Foundation Chair in Translational Neuroengineering, Center for Neuroprosthetics and Institute of Bioengineering, School of Engineering, École Polytechnique Fédérale de Lausanne (EPFL), Lausanne, Switzerland, ${ }^{2}$ The BioRobotics Institute and Department of Excellence in Al and Robotics, Scuola Superiore Sant'Anna, Pisa, Italy, ${ }^{3}$ Laboratory for Neuroengineering, Department of Health Sciences and Technology, Institute for Robotics and Intelligent Systems, ETH Zürich, Zurich, Switzerland, ${ }^{4}$ Department of Excellence in Robotics and Al, Scuola Superiore Sant'Anna, Pisa, Italy, ${ }^{5}$ Institute of Neurology, Catholic University of The Sacred Heart, Policlinic A. Gemelli Foundation, Rome, Italy, ${ }^{6}$ Laboratory for Biomedical Microtechnology, Department of Microsystems Engineering-IMTEK, Bernstein Center, BrainLinks-BrainTools Cluster of Excellence, University of Freiburg, Freiburg im Breisgau, Germany, ${ }^{7}$ Area of Neuroscience, IRCCS San Raffaele Pisana, Rome, Italy
\end{abstract}

\section{OPEN ACCESS}

Edited by:

Manolo Garabini,

University of Pisa, Italy

Reviewed by:

Vincenzo Catrambone,

University of Pisa, Italy

Salvatore Andrea Pullano,

University Magna Graecia

of Catanzaro, Italy

*Correspondence:

Silvestro Micera

silvestro.micera@epfl.ch

+ORCID:

Giacomo Valle

orcid.org/0000-0002-2637-8007

Specialty section:

This article was submitted to

Bionics and Biomimetics,

a section of the journal

Frontiers in Bioengineering and

Biotechnology

Received: 15 July 2019

Accepted: 18 March 2020

Published: 03 April 2020

Citation:

Valle G, D'Anna E, Strauss I, Clemente F, Granata G, Di lorio R, Controzzi M, Stieglitz T, Rossini PM,

Petrini FM and Micera S (2020) Hand Control With Invasive Feedback Is Not Impaired by Increased Cognitive

Front. Bioeng. Biotechnol. 8:287. doi: 10.3389/fbioe.2020.00287
Recent experiments have shown that neural stimulation can successfully restore sensory feedback in upper-limb amputees improving their ability to control the prosthesis. However, the potential advantages of invasive sensory feedback with respect to noninvasive solutions have not been yet identified. Our hypothesis was that a difference would appear when the subject cannot focus all the attention to the use of the prosthesis, but some additional activities require his/her cognitive attention, which is a quite common situation in real-life conditions. To verify this hypothesis, we asked a trans-radial amputee, equipped with a bidirectional hand prosthesis, to perform motor tasks also in combination with a cognitive task. Sensory feedback was provided via intraneural (invasive) or electro-tactile (non-invasive) stimulation. We collected also data related to self-confidence. While both approaches were able to significantly improve the motor performance of the subject when no additional cognitive effort was asked, the manual accuracy was not affected by the cognitive task only when intraneural feedback was provided. The highest self-confidence was obtained when intraneural sensory feedback was provided. Our findings show that intraneural sensory feedback is more robust to dual tasks than non-invasive feedback. This is the first direct comparison between invasive and non-invasive approaches for restoring sensory feedback and it could suggest an advantage of using invasive solutions.

Clinical Trial Registration: www.ClinicalTrials.gov, identifier NCT02848846.

Keywords: neural sensory feedback, superficial sensory feedback, upper limb amputees, prosthesis, cognitive load, neural interfaces, electrical stimulation

\section{INTRODUCTION}

The loss of a hand affects persons' quality of life (Meyer, 2003). Several clinical solutions have been provided compared to the first manufactured prostheses, developing more dexterous artificial hands (Belter et al., 2013). Nevertheless, the lack of sensory information flow from the missing hand is still among the reasons for prosthesis underuse (Biddiss and Chau, 2007). To this aim, several invasive (Raspopovic et al., 2014; Tan et al., 2014; Davis et al., 2016; Petrini et al., 2018) 
and non-invasive (Marasco et al., 2018; Osborn et al., 2018) technologies have been developed to restore sensory feedback in upper limb amputees. Different implantable peripheral interfaces have been shown to efficiently stimulate the sensory nerves restoring natural sensations (Raspopovic et al., 2014; Tan et al., 2014; Valle et al., 2018) optimally integrated (Risso et al., 2019), improving prosthesis control (Tan et al., 2014; Petrini et al., 2018; Valle et al., 2018) and prosthesis embodiment (Valle et al., 2018), diminishing phantom limb pain (Petrini et al., 2018) also in chronic, long-lasting applications (Tan et al., 2014; Petrini et al., 2018).

However, since daily activities are frequently performed in a "dual-task" paradigm condition (i.e., holding a beer while reading a book) (Land et al., 1999), the execution of motor tasks with a bidirectional prosthesis should be assessed in combination with tasks increasing the cognitive load for the user. To accomplish this dual paradigm the user cannot (or should not) focus all the attention to the use of the prosthesis. This is a very important issue but, so far, the impact of cognitive efforts on the efficacy of the ongoing artificial sensory feedback has not been investigated. Here, we evaluate the effect of using a bidirectional hand prosthesis with intraneural, with noninvasive electrotactile or with no sensory feedback in a dualtask paradigm in which subjects can use the visual feedback (Figure 1). A trans-radial amputee was recruited and implanted with transversal intrafascicular multichannel electrodes [TIME (Petrini et al., 2018; Valle et al., 2018)]. In particular, we assessed the short-term memory capacity [STC or memory span (Lovett et al., 2000; Jones and Macken, 2015)] while the patient was performing a motor task [Virtual Eggs Test (Clemente et al., 2016)] requiring manual accuracy and dexterity in three different sensory feedback conditions (Figure 1). We provided to the amputee: (i) intraneural sensory feedback (IF) delivering stimulation trains through an implanted TIME in the ulnar nerve, or (ii) electrotactile sensory feedback (SF) delivering electrical stimulation through a surface electrode placed on the residual arm skin or (iii) no sensory feedback (NF). We measured patient's performance reporting memory span, manual accuracy and manual dexterity achieved during the dual-task. Results indicate that only intraneural sensory feedback could allow to achieve a robust improvement in motor performance, maintaining a high STC, also in case of increased cognitive load.

\section{METHODS}

\section{Patient Recruitment and Surgical Procedures}

A single patient participated in this study after providing her informed consent: a right-handed 54-year-old female with a distal two-thirds of the left forearm trans-radial amputation incurred 2 years prior to the study.

Briefly, the subject was implanted with four TIMEs, two in the median nerve and two in the ulnar nerve (above the elbow). Overall, 56 active sites were available (14 per electrode). They were implanted on June 24th 2017. The explant of the electrodes was executed on December 16th 2017. The electrode cable segments were located in subcutaneous pockets, externalized (and secured with sutures and subcutaneous strain release loops) in order to be available for the transcutaneous connection with a neural stimulator (Petrini et al., 2018) (Ripple LLC).

\section{Prosthesis Movement Control}

The bidirectional control setup is described in detail in Valle et al. (2018). Briefly, for prosthesis control, surface electromyographic signals (sEMG) were acquired from the forearm muscles and decoded using a k-NN classifier (3 classes: close, open and rest). The control algorithm was the same in each condition. The decoded movement was sent to a prosthetic hand (IH2 Azzurra, Prensilia, Italy), equipped with tension and position sensors in each digit. Based on the recorded position and tension information, stimulation pulses were delivered through the four TIME electrodes.

\section{Neural and Electrotactile Sensory Feedback}

Intraneural tactile feedback was delivered using the same setup described in Valle et al. (2018). After an extensive mapping phase, as described in Petrini et al. (2018) and Valle et al. (2018) we identified the optimal stimulation parameters for each

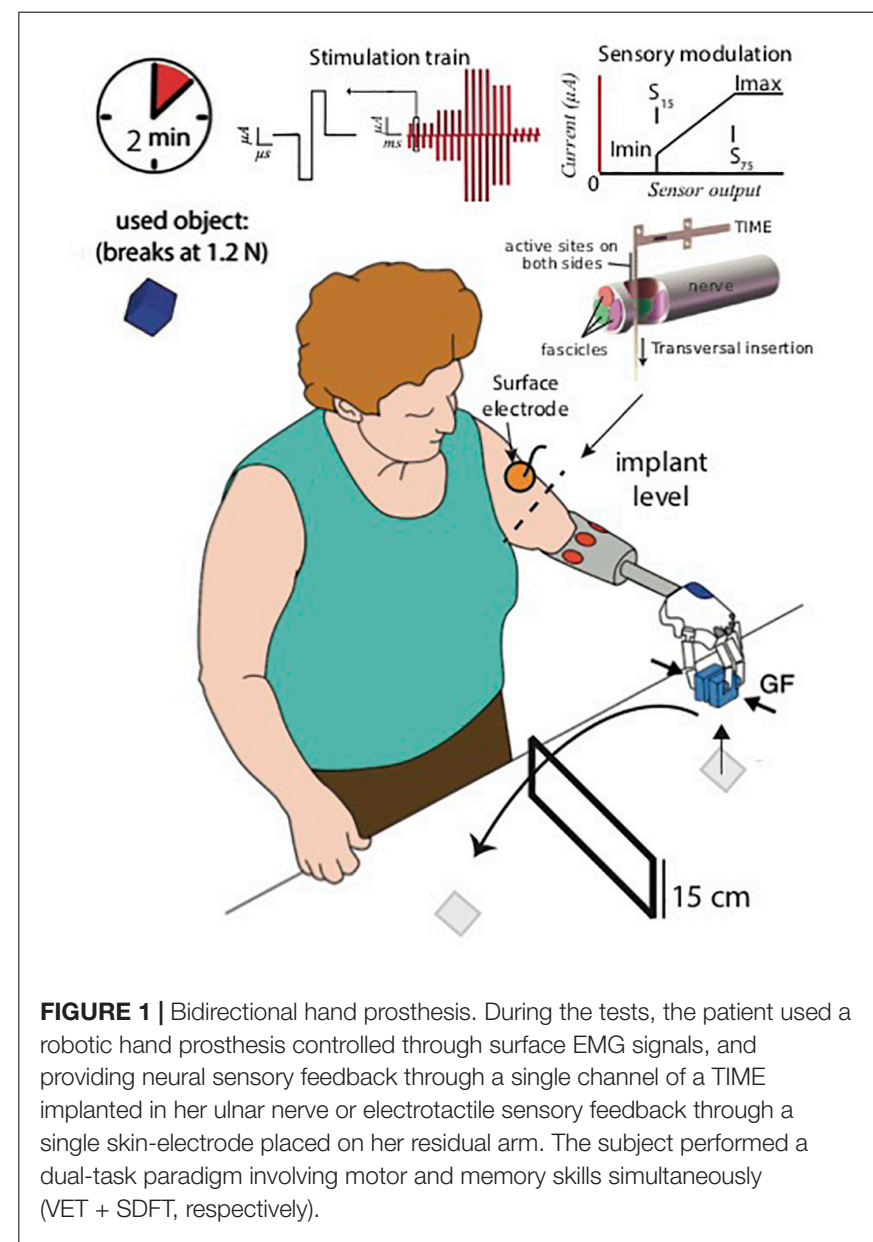




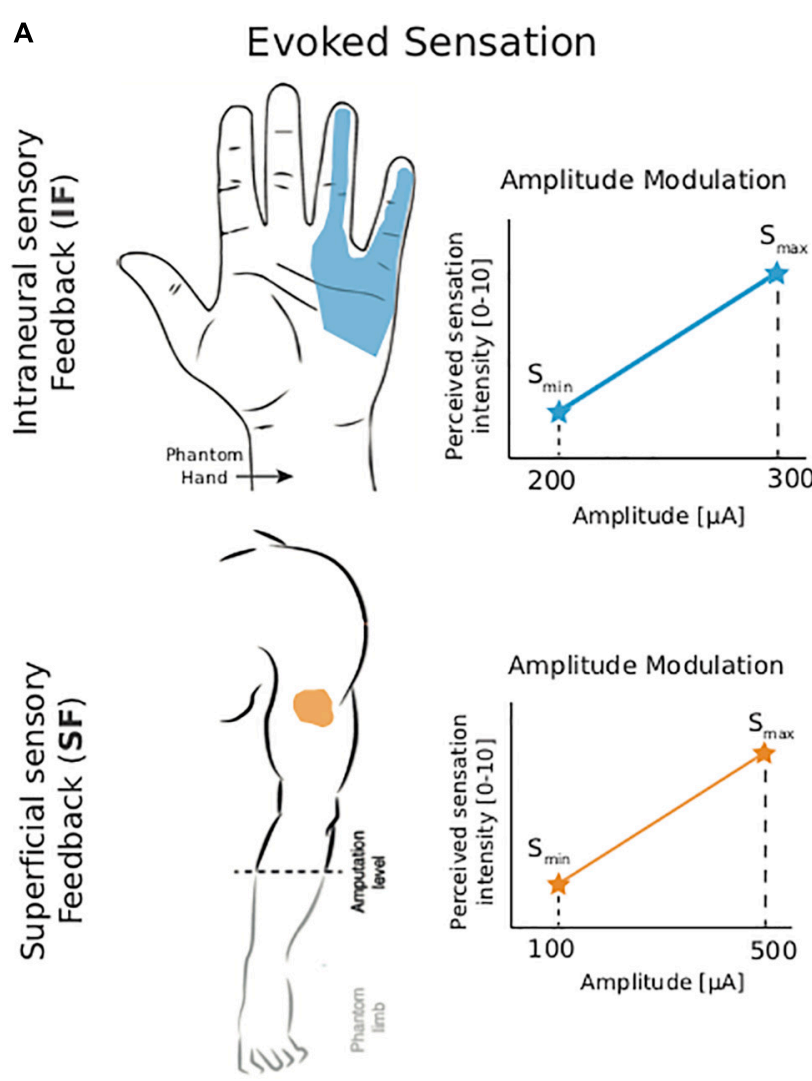

\section{B Induced sensations $\&$ stimulation parameters}

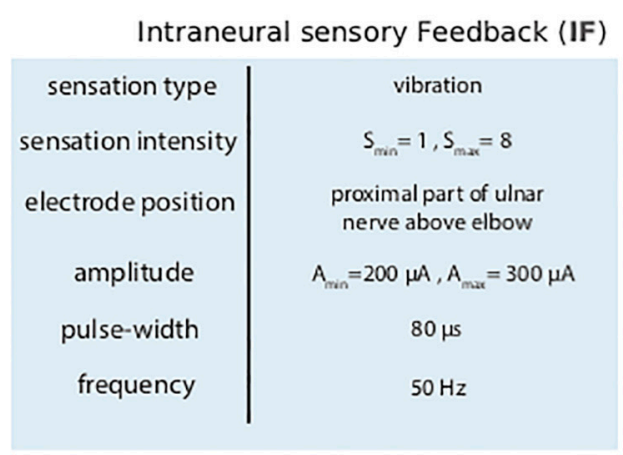

\section{Superficial sensory Feedback (SF)}

\begin{tabular}{c|c} 
sensation type & electricity \\
sensation intensity & $\mathrm{S}_{\min }=1, \mathrm{~S}_{\max }=8$ \\
electrode position & on the skin of the left arm \\
amplitude & $\mathrm{A}_{\min }=100 \mu \mathrm{A}, \mathrm{A}_{\max }=500 \mu \mathrm{A}$ \\
pulse-width & $200 \mu \mathrm{s}$ \\
frequency & $50 \mathrm{~Hz}$
\end{tabular}

FIGURE 2 | Sensory feedback. (A) Locations of the elicited sensation when Intraneural sensory Feedback (IF) and Superficial sensory Feedback were provided. Both encoding strategies exploited amplitude modulation. (B) Induced sensation and stimulation parameters were reported for intraneural stimulation (IF) and superficial electrotactile stimulation (SF).

channel, such as perceptual threshold stimulation charge and pain thresholds. Stimulation frequency was always fixed at $50 \mathrm{~Hz}$ as in previous studies (Raspopovic et al., 2014), and injected current levels were always below the chemical safety limit of $120 \mathrm{nC}$ (Petrini et al., 2018). To achieve sensation modulation, the injected charge was modulated by changing the injected current amplitude. Rectangular, biphasic stimulation pulse amplitudes were modulated between 200 and $300 \mu \mathrm{A}$ with a fixed pulse width of $80 \mu \mathrm{s}$. In all cases, the resulting sensation was described as pressure or vibration referred to most of the ulnar innervation area (Figure 2A). The patient reported a direct proportional correlation between the amplitude of the stimulation injected and the intensity of the evoked sensation, producing a very limited increase of the extent of the area of the elicited sensations [details reported in Petrini et al. (2018)].

For a non-invasive approach an electrotactile stimulation was used placing a surface electrode on the arm. Stimulation was delivered as square charge balanced biphasic pulse trains with a fixed frequency [ $50 \mathrm{~Hz}$ as in D'Anna et al. (2017)], in such a way as to elicit only in loco sensation under the electrode. Perceptual thresholds and pain thresholds were identified using the same approach used for intraneural stimulation and described in Petrini et al. (2018). The amplitude used varied between $100 \mu \mathrm{A}$ and $500 \mu \mathrm{A}$, with a pulse width duration of $200 \mu$ s (Figure 2A).
Before these experiments the patient already exploited both IF and $\mathrm{SF}$ in a bidirectional hand prosthesis during extensive tests (Petrini et al., 2018; D’Anna et al., 2019).

\section{Dual-Task Paradigm}

In the dual-task paradigm, the patient was asked to perform the Virtual Eggs Test (VET) and the Span Digit Forward Test [SDFT (Blackburn and Benton, 1957)] simultaneously. The VET is a recently proposed test for sensorimotor assessment (Clemente et al., 2016). During the VET, the patient, wearing the prosthesis, was instructed to transfer the fragile blocks presented in front of her from one side to the other over a $15 \mathrm{~cm}$ tall wall as fast as possible and without breaking them (Figure 1). The performances were measured by the number of transferred (broken and unbroken) blocks (gross manual dexterity, D) and the number of transferred unbroken blocks over the total number of blocks (manual accuracy, A) during 2-min trials. In this work, the virtual eggs would break when grasped with a grip force larger than 1.2 N, which was determined in Petrini et al. (2018). This motor task was previously presented and used to assess motor performance in transradial amputees (Petrini et al., 2018; Valle et al., 2018).

During the VET, the patient was asked to repeat digits in the order in which they were read out by the experimenter (SDFT). 
The digits were asked in an even tone, at approximately the rate of one digit per second. The patient's memory span was defined as the maximum length of the digit lists of which the patient recalled correctly (Lezak et al., 2012). The memory span at rest condition was also reported as a baseline. The response time, defined as the time requested by the patient to repeat the entire digits list, was collected in each condition using a chronometer. The patient performed 15 trials for each condition (IF, SF, and NF). Each condition was tested in a different day by the patient after several tests for each of them (Petrini et al., 2018; D'Anna et al.,

\begin{tabular}{|l|}
\hline $\mathrm{F}=$ Intraneural sensory Feedback \\
$\mathrm{SF}=$ Superficial sensory Feedback \\
$\mathrm{NF}=$ No sensory Feedback \\
$\mathrm{C}-\mathrm{ON}=$ Cognitive load ON \\
$\mathrm{C}-\mathrm{OFF}=$ Cognitive load OFF
\end{tabular}

A

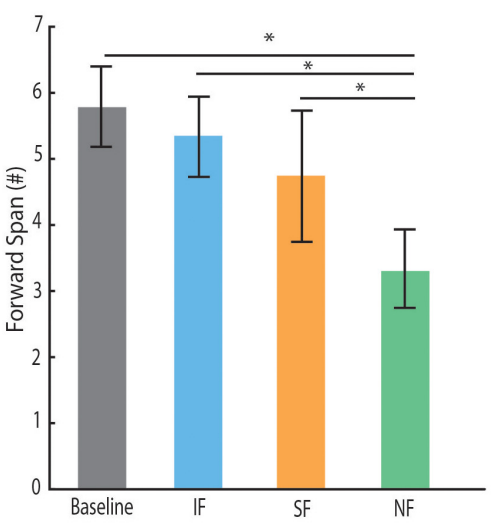

C

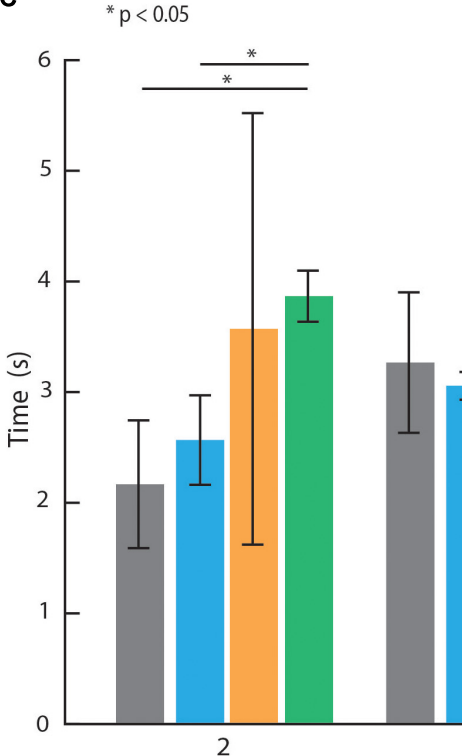

\section{Cognitive Dual Task (Span Digit Forward Test during Virtual Eggs Test)}

B

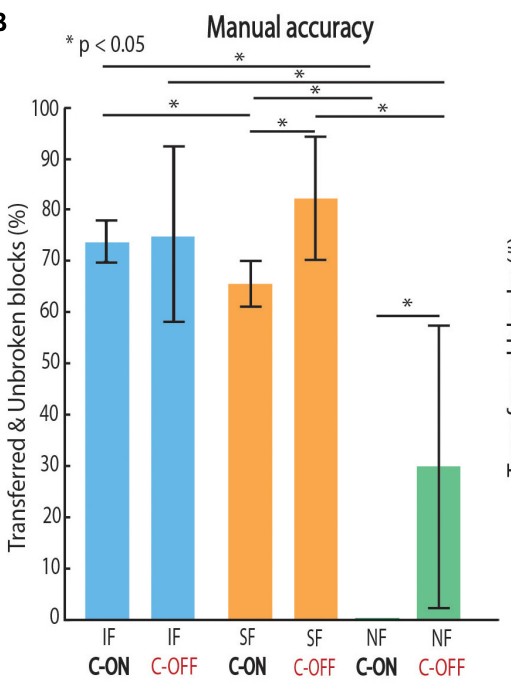

Response time

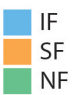

Gross manual dexterity

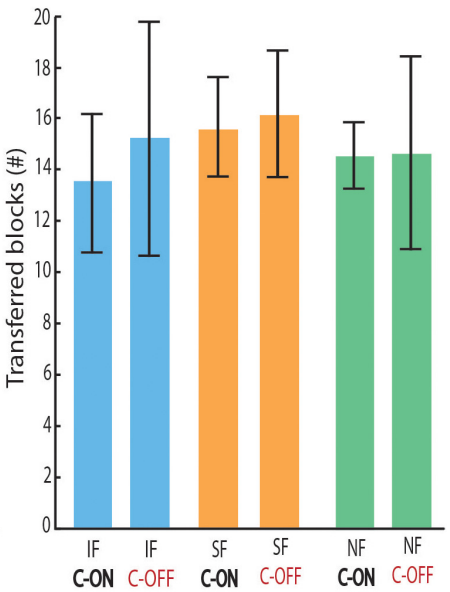

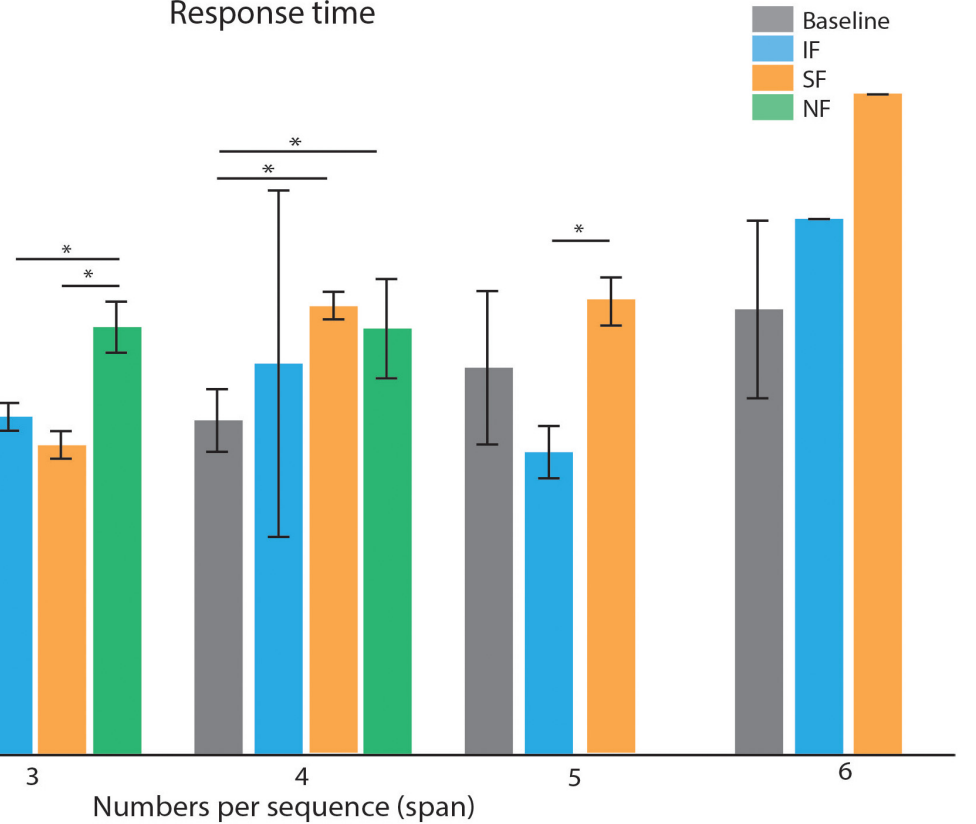

FIGURE 3 | Dual-task: motor control and short-term memory assessment. (A) Memory digit spans according to the different conditions are presented. Baseline was acquired as control value. (B) Performances are evaluated as manual accuracy (number of unbroken and transferred blocks over total transferred blocks) and manual dexterity (number of total transferred blocks). (C) Time to recall all the digits sequence was collected in each condition. All data are reported as mean values \pm standard deviations. A span of 6 was reached only once in SF and once in IF (error bars without std). Friedman test, with Tukey-Kramer correction for multiple groups of data when requested, was performed. We performed 5 repetitions $\times 15$ feedback conditions (Intraneural Feedback - IF, Superficial Feedback SF and No Feedback - NF) $\times 2$ cognitive conditions (with Cognitive task C-ON and without Cognitive task C-OFF). 
2019). The VET performances were evaluated in each stimulation condition with (C-ON) and without (C-OFF) SDFT (Figure 3B).

For each trial set, after the task we asked the subject to rate her confidence in her ability to perform the task, on a scale of 0 to 10 (Schiefer et al., 2018).

\section{Statistical Analysis}

All data were analyzed using MATLAB (R2016a, The MathWorks, Natick, MA, United States). All statistics were performed using the available built-in functions. The data were not normally distributed, the $p$-values of the KolmogorovSmirnov test were always $>0.1$. Thus, the Friedman test, with Tukey-Kramer correction for multiple groups of data when requested, was performed. All reported $p$-values resulting from the Friedman test (p) measure the significance of the statistic. The number of repetitions for each experiment is reported in the corresponding figure captions.

\section{Data Availability Statement}

The datasets (also protocol and statistical analysis plan) generated during and/or analyzed during the current study are available from the corresponding author on reasonable request.

\section{Standard Protocol Approvals, Registrations, and Patient Consents}

Ethical approval was obtained by the Institutional Ethics Committees of Policlinic Agostino Gemelli at the Catholic University, Rome, Italy, where the surgery was performed. The protocol was also approved by the Italian Ministry of Health. Written informed consent and authorization for disclosure were signed by the patient. The clinical trial's registration number on the online platform (www.ClinicalTrials.gov) is NCT02848846.

\section{RESULTS}

Both the invasive and non-invasive sensory feedback approaches were able to provide meaningful sensations to the patient (see Figures 2A,B). These sensations were then used by the subject during the experiments.

In order to assess the effect of adding sensory feedback to a hand prosthesis in a dual-task paradigm, we evaluated the memory span among the tested conditions. In particular, we found that the memory span was statistically lower Span $_{\text {baseline }}=5.7 \pm 0.6, \operatorname{Span}_{\mathrm{IF}}=5.5 \pm 0.6, \operatorname{Span}_{\mathrm{SF}}=4.6 \pm 1.3$, Span $_{\mathrm{NF}}=3.3 \pm 0.7$ ), when no sensory feedback was provided compared to baseline, IF and SF (Friedman test, $p<0.01$; Figure 3A). On the contrary, when adding a sensory feedback, no change of the memory span with respect to baseline (Friedman test, $p>0.05$; Figure 3A) was found. Looking at the functional performance estimation done by VET, firstly, the patient increased the percentage of unbroken and transferred blocks (manual accuracy, A) when using the sensory feedback $\left(\mathrm{A}_{\mathrm{IF}, \mathrm{C}-\mathrm{OFF}}=74.3 \pm 4.6 \%, \mathrm{~A}_{\mathrm{SF}, \mathrm{C}-\mathrm{OFF}}=83.3 \pm 12.7 \%\right.$ and $\mathrm{A}_{\mathrm{NF}, \mathrm{C}-\mathrm{OFF}}=30.6 \pm 27.4 \%$ ), maintaining similar number of transferred blocks in total (gross manual dexterity, D) $\left(\mathrm{D}_{\mathrm{IF}, \mathrm{C}-\mathrm{OFF}}=15 \pm 4, \mathrm{D}_{\mathrm{SF}, \mathrm{C}-\mathrm{OFF}}=16 \pm 2\right.$ and
$\mathrm{D}_{\mathrm{NF}, \mathrm{C}-\mathrm{OFF}}=14.5 \pm 3.5$ ). Considering the gross manual dexterity, during the dual-task it was similar $\left(\mathrm{D}_{\mathrm{IF}, \mathrm{C}-\mathrm{ON}}=13.8 \pm 2.2\right.$, $\mathrm{D}_{\mathrm{SF}, \mathrm{C}-\mathrm{ON}}=15.5 \pm 1.5$ and $\left.\mathrm{D}_{\mathrm{NF}, \mathrm{C}-\mathrm{ON}}=14.3 \pm 0.5 p>0.05\right)$ to the one achieved during VET alone (without SDFT, C-OFF) in all conditions (Figure 3B, left). On the contrary, the patient's manual accuracy was affected when a second task (with SDFT, $\mathrm{C}-\mathrm{ON}$ ) was added to VET. In particular, in NF condition the patient was totally unable to move unbroken blocks over the barrier showing a significant decrement of the manual accuracy (Friedman test, $p<0.01$ ). When SF was provided in the dual-task paradigm, the performance significantly decreased $(-17.3 \%$, $p<0.05$ ) showing that the cognitive effort was still high. Finally, in IF condition, the performance was not statistically different in the dual-task compared to the single task (Friedman test, $p>0.05$ ), indicating that the neural sensory feedback was easier to integrate into the sensorimotor control. Furthermore, the response time (Figure 3C) in IF condition did not show any difference with the one measured at the baseline (Friedman test, $p>0.05$ in all cases) unlike in SF and NF conditions.

Looking at the results regarding the self-confidence (Figure 4), during IF the score $(7.7 \pm 0.48)$ was significantly greater than in SF $(5.6 \pm 0.51)$ and in NF $(3.4 \pm 0.51)$ (Friedman test, $p>0.05)$. Confidence was also statistically higher when SF was provided compared to NF condition (Friedman test, $p>0.05$ ).

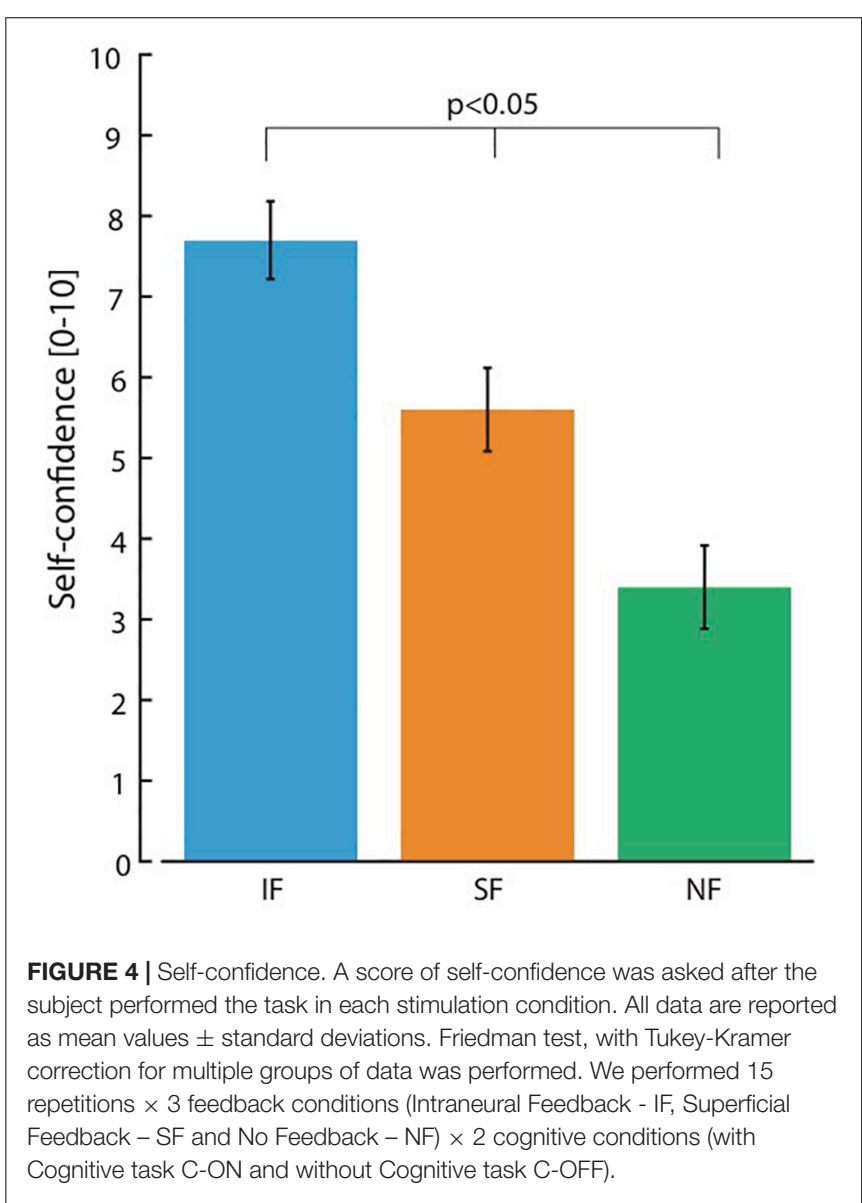




\section{DISCUSSION}

Providing a tactile sensory feedback [superficial (SF) or intraneural (IF)] from sensors embedded in a hand prosthesis, while performing a motor task (VET), leads to maintain the memory span and count speed similar to the baseline (no motor task). It means that the short-term memory (STM) was overloaded by the dual-task in NF condition. The patient could not control the prosthesis, regulate the grip force relying only on visual feedback and recall the numbers simultaneously. Indeed, in NF condition also the performance in manual accuracy during the VET diminished significantly. Interestingly, the variability of the manual accuracy in NF condition was higher than in IF and SF. This was probably due to the poor reliability of the visual feedback to extract tactile information from the environment (e.g., grip force). Our results confirmed that the lack of a sensory inputs is reflected in increased cognitive effort (Williams et al., 2006; Raveh et al., 2018) during a dualtask. Looking at the sensory feedback type provided during the task, IF performed better than SF allowing to maintain high manual accuracy, with similar dexterity, even in the dualtask. Indeed, the manual accuracy in IF was statistically higher than SF only in C-ON condition. This evidence shows that the performance of an invasive or non-invasive technology is different when a daily activity (i.e., a task requiring more than one action simultaneously) is simulated. It could suggest that a more somatotopic, homologous and selective sensory feedback could be optimally exploited, and it could help more in daily living situations. Also, the response time in IF condition was on average lower than in NF and SF. This could indicate that the neural sensory feedback was easier to process, being more natural, showing a better integration into the sensorimotor control. The difference between IF and SF may be due to the different somatotopy of the sensation provided (in loco for SF and referred from the phantom hand in IF) and the sensation type (electricity for SF and vibration for IF). A somatotopic non-invasive stimulation (D'Anna et al., 2017; Osborn et al., 2018) should be tested and compared to verify these hypotheses. Further experiments are necessary to investigate these dependencies with more subjects and while performing different tasks also considering practice effects.

When a sensory feedback was provided, the subject had a higher self-confidence while performing the task. This result was consistent with previously presented experiments (Schiefer et al., 2018), in which transradial amputees were asked to give a score on their self-confidence while they were exploiting a bidirectional hand prosthesis in object identification tasks with and without the invasive sensory feedback. Interestingly also in our study, the subject was more confident when IF was provided respect to SF. This could be an ulterior evidence that a sensory feedback more somatotopic and more natural is better integrated and processed by the subject. In addition, the modal congruence of the stimulation-elicited percepts to the subject's expectations may have influenced the relative weightings of the information.

Our results on a single patient need to be repeated in a large population of hand amputees in order to compare IF and SF techniques.
Moreover, recently, more biomimetic approaches were developed for restoring tactile sensory feedback (Valle et al., 2018), mimicking more closely the physiological behavior of natural sensors in the skin. Since in this work we used only the linear modulation of the current amplitude as a function of the prosthesis sensor readouts, it would be also interesting to investigate what happens with other encoding paradigms (Okorokova et al., 2018).

We believe that our findings support the hypothesis that intraneural stimulation providing sensory feedback to trans-radial amputee, could be effectively integrated into the sensorimotor control. Indeed, it improves the manual accuracy and dexterity of a hand prosthesis even when the motor control task is executed simultaneously with a cognitive task increasing the cognitive load.

\section{DATA AVAILABILITY STATEMENT}

The datasets generated for this study are available on request to the corresponding author.

\section{ETHICS STATEMENT}

The studies involving human participants were reviewed and approved by Policlinico Gemelli and Italian Ministry of Research. The patients/participants provided their written informed consent to participate in this study. During the entire duration of our study, all experiments were conducted in accordance with EU guidelines and regulations.

\section{AUTHOR CONTRIBUTIONS}

GV, FC, MC, FP, and SM designed the experiment. GV, IS, and ED'A collected the data. GV analyzed the data, made the figures and drafted the manuscript. FC, MC, IS, FP, ED'A, TS, PR, and SM edited and revised the manuscript. GV, ED'A, and IS developed the software and the overall system integration. GG, $\mathrm{RD}$, and PR selected the patient and were responsible for all the clinical aspects of the study. TS developed the TIME electrodes.

\section{FUNDING}

This work was supported by the EU Grant FP7-611687 NEBIAS (NEurocontrolled BIdirectional Artificial upper limb and handprosthesis), the Swiss National Science Foundation through the National Centre of Competence in Research (NCCR) Robotics and the project CHRONOS. The funder had no role in the experimental design, analysis, or manuscript preparation or submission.

\section{ACKNOWLEDGMENTS}

The authors are grateful to the participant for the experimentation. 


\section{REFERENCES}

Belter, J. T., Segil, J. L., Dollar, A. M., and Weir, R. F. (2013). Mechanical design and performance specifications of anthropomorphic prosthetic hands: a review. J. Rehabil. Res. Dev. Wash. 50, 599-618.

Biddiss, E. A., and Chau, T. T. (2007). Upper limb prosthesis use and abandonment: a survey of the last 25 years. Prosthet. Orthot. Int. 31, 236-257. doi: 10.1080/ 03093640600994581

Blackburn, H. L., and Benton, A. L. (1957). Revised administration and scoring of the digit span test. J. Consult. Psychol. 21, 139-143. doi: 10.1037/h0047235

Clemente, F., D’Alonzo, M., Controzzi, M., Edin, B. B., and Cipriani, C. (2016). Non-invasive, temporally discrete feedback of object contact and release improves grasp control of closed-loop myoelectric transradial prostheses. IEEE Trans. Neural Syst. Rehabil. Eng. 24, 1314-1322. doi: 10.1109/TNSRE.2015. 2500586

D’Anna, E., Petrini, F. M., Artoni, F., Popovic, I., Simanić, I., Raspopovic, S., et al. (2017). A somatotopic bidirectional hand prosthesis with transcutaneous electrical nerve stimulation based sensory feedback. Sci. Rep. 7:10930. doi: 10. 1038/s41598-017-11306-w

D’Anna, E., Valle, G., Mazzoni, A., Strauss, I., Iberite, F., Patton, J., et al. (2019). A closed-loop hand prosthesis with simultaneous intraneural tactile and position feedback. Sci. Robot. 4:eaau8892. doi: 10.1126/scirobotics.aau8892

Davis, T. S., Wark, H. A. C., Hutchinson, D. T., Warren, D. J., O’Neill, K., Scheinblum, T., et al. (2016). Restoring motor control and sensory feedback in people with upper extremity amputations using arrays of 96 microelectrodes implanted in the median and ulnar nerves. J. Neural Eng. 13:036001. doi: 10. 1088/1741-2560/13/3/036001

Jones, G., and Macken, B. (2015). Questioning short-term memory and its measurement: why digit span measures long-term associative learning. Cognition 144, 1-13. doi: 10.1016/j.cognition.2015.07.009

Land, M., Mennie, N., and Rusted, J. (1999). The roles of vision and eye movements in the control of activities of daily living. Perception 28, 1311-1328. doi: 10.1068/ p2935

Lezak, M. D., Howieson, D. B., Bigler, E. D., and Tranel, D. (2012). Neuropsychological Assessment. Oxford: Oxford University Press.

Lovett, M. C., Daily, L. Z., and Reder, L. M. (2000). A source activation theory of working memory: cross-task prediction of performance in ACT-R. Cogn. Syst. Res. 1, 99-118. doi: 10.1016/S1389-0417(99)00012-11

Marasco, P. D., Hebert, J. S., Sensinger, J. W., Shell, C. E., Schofield, J. S., Thumser, Z. C., et al. (2018). Illusory movement perception improves motor control for prosthetic hands. Sci. Transl. Med. 10:aao6990. doi: 10.1126/scitranslmed. aao6990

Meyer, T. M. (2003). Psychological aspects of mutilating hand injuries. Hand Clin. 19, 41-49. doi: 10.1016/s0749-0712(02)00056-2

Okorokova, E., He, Q., and Bensmaia, S. J. (2018). Biomimetic encoding model for restoring touch in bionic hands through a nerve interface. J. Neural Eng. 15:066033. doi: 10.1088/1741-2552/aae398

Osborn, L. E., Dragomir, A., Betthauser, J. L., Hunt, C. L., Nguyen, H. H., Kaliki, R. R., et al. (2018). Prosthesis with neuromorphic multilayered e-dermis perceives touch and pain. Sci. Robot. 19:eaat3818. doi: 10.1126/scirobotics. aat 3818
Petrini, F. M., Valle, G., Strauss, I., Granata, G., Di Iorio, R., D’Anna, E., et al. (2018). Six-months assessment of a hand prosthesis with intraneural tactile feedback. Ann. Neurol. 85, 137-154. doi: 10.1002/ana. 25384

Raspopovic, S., Capogrosso, M., Petrini, F. M., Bonizzato, M., Rigosa, J., Di Pino, G., et al. (2014). Restoring natural sensory feedback in real-time bidirectional hand prostheses. Sci. Transl. Med. 6:222ra19. doi: 10.1126/scitranslmed. 3006820

Raveh, E., Friedman, J., and Portnoy, S. (2018). Evaluation of the effects of adding vibrotactile feedback to myoelectric prosthesis users on performance and visual attention in a dual-task paradigm. Clin. Rehabil. 32, 1308-1316. doi: 10.1177/ 0269215518774104

Risso, G., Valle, G., Iberite, F., Strauss, I., Stieglitz, T., Controzzi, M., et al. (2019). Optimal integration of intraneural somatosensory feedback with visual information: a single-case study. Sci. Rep. 9:7916. doi: 10.1038/s41598-01943815-43811

Schiefer, M. A., Graczyk, E. L., Sidik, S. M., Tan, D. W., and Tyler, D. J. (2018). Artificial tactile and proprioceptive feedback improves performance and confidence on object identification tasks. PLoS One 13:e0207659. doi: 10.1371/ journal.pone.0207659

Tan, D. W., Schiefer, M. A., Keith, M. W., Anderson, J. R., Tyler, J., and Tyler, D. J. (2014). A neural interface provides long-term stable natural touch perception. Sci. Transl. Med. 6:257ra138. doi: 10.1126/scitranslmed.30 08669

Valle, G., Mazzoni, A., Iberite, F., D’Anna, E., Strauss, I., Granata, G., et al. (2018). Biomimetic intraneural sensory feedback enhances sensation naturalness, tactile sensitivity, and manual dexterity in a bidirectional prosthesis. Neuron 100:37.e-45.e. doi: 10.1016/j.neuron.2018. 08.033

Williams, R. M., Turner, A. P., Orendurff, M., Segal, A. D., Klute, G. K., Pecoraro, J., et al. (2006). Does having a computerized prosthetic knee influence cognitive performance during amputee walking? Arch. Phys. Med. Rehabil. 87, 989-994. doi: 10.1016/j.apmr.2006.03.006

Conflict of Interest: FP and SM hold shares of "SensArs Neuroprosthetics Sàrl," a start-up company dealing with commercialization of neurocontrolled artificial limbs. MC and FC hold shares of "Prensilia," a start-up company commercializing robotic hands and assessment tools.

The remaining authors declare that the research was conducted in the absence of any commercial or financial relationships that could be construed as a potential conflict of interest.

Copyright (c) 2020 Valle, D’Anna, Strauss, Clemente, Granata, Di Iorio, Controzzi, Stieglitz, Rossini, Petrini and Micera. This is an open-access article distributed under the terms of the Creative Commons Attribution License (CC BY). The use, distribution or reproduction in other forums is permitted, provided the original author(s) and the copyright owner(s) are credited and that the original publication in this journal is cited, in accordance with accepted academic practice. No use, distribution or reproduction is permitted which does not comply with these terms. 